\title{
Reevaluating the sensory recruitment model by manipulating crowding in visual working memory representations
}

\author{
Harun Yörük ${ }^{1}$ - Lindsay A. Santacroce ${ }^{1} \cdot$ Benjamin J. Tamber-Rosenau ${ }^{1}$ \\ Published online: 8 June 2020 \\ (C) The Psychonomic Society, Inc. 2020
}

\begin{abstract}
The prominent sensory recruitment model argues that visual working memory (WM) is maintained via representations in the same early visual cortex brain regions that initially encode sensory stimuli, either in the identical neural populations as perceptual representations or in distinct neural populations. While recent research seems to reject the former (strong) sensory recruitment model, the latter (flexible) account remains plausible. Moreover, this flexibility could explain a recent result of high theoretical impact (Harrison \& Bays, The Journal of Neuroscience, 38 (12), 3116-3123, 2018) - a failure to observe interactions between items held in visual WM - that has been taken to reject the sensory recruitment model. Harrison and Bays (The Journal of Neuroscience, 38 (12), 3116-3123, 2018) tested the sensory recruitment model by comparing the precision of memoranda in radially and tangentially oriented memory arrays. Because perceptual visual crowding effects are greater in radial than tangential arrays, they reasoned that a failure to observe such anisotropy in WM would reject the sensory recruitment model. In the present Registered Report or Replication, we replicated their study with greater sensitivity and extended their task by controlling a potential strategic confound. Specifically, participants might remap memory items to new locations, reducing interactions between proximal memoranda. To combat remapping, we cued participants to report either a memory item or its precise location - with this report cue presented only after a memory maintenance period. Our results suggest that, similar to visual perceptual crowding, location-bound visual memoranda interact with one another when remapping is prevented. Thus, our results support at least a flexible form of the sensory recruitment model.
\end{abstract}

Keywords Visual working memory $\cdot$ Crowding $\cdot$ Cortical representation $\cdot$ Sensory recruitment

A decade ago, two foundational papers (Harrison \& Tong, 2009; Serences, Ester, Vogel, \& Awh, 2009) used "information-based" (c.f., Kriegeskorte, Goebel, \& Bandettini, 2006) multivariate analyses of functional neuroimaging data to demonstrate that the contents of visual working memory (VWM) could be decoded from the spatial pattern of activation in visual cortex even in the absence of sustained activation. These papers provided neuroscientific support for the sensory recruitment model - the notion that working memory representations are supported by ongoing activity in the very same cortical machinery that

Electronic supplementary material The online version of this article (https://doi.org/10.3758/s13423-020-01757-0) contains supplementary material, which is available to authorized users.

Benjamin J. Tamber-Rosenau

bjtamber-rosenau@uh.edu; bjtrbjtr@gmail.com

1 Department of Psychology, University of Houston, Heyne Building Room 126, 3695 Cullen Blvd, Houston, TX 77204, USA supports initial perceptual representations. A recent study (Harrison \& Bays, 2018) seriously undermined this support by testing whether VWM and perceptual vision share a feature of their representations thought to stem from the retinotopic configuration of early visual cortex - greater interactions between nearby items when they are arranged radially compared to tangentially. Because they observed that VWM did not share this property, they argued that the representational basis of VWM must not be dependent on the same retinotopic representations as perceptual vision. In the present Registered Report or Replication, we argue that the failure to observe a radial/tangential anisotropy in VWM could stem from the flexibility of VWM to represent only task-relevant information. We reasoned that if we required participants to remember the locations of memoranda, as is typical in studies of VWM, we might then observe the elusive radial/tangential crowding anisotropy - even for VWM items. If so, the sensory recruitment model could no longer be dismissed on the basis of the results of Harrison and Bays (2018). 
The sensory recruitment model is extremely appealing because it does not require a distinct VWM copy of perceptual representations to reside outside of sensory cortex, and it is hard to conceive of how such a second-copy system could provide the high fidelity of VWM without duplicating much of the sensory cortex of the brain in another cortical location. Additionally, though there is sustained neural activity in the prefrontal cortex during VWM (Courtney, Ungerleider, Keil, \& Haxby, 1997; Levy \& Goldman-Rakic, 1999, 2000), by the early 2000s the view emerged that such prefrontal activity reflects a biasing signal to protect sensory cortical representations (Curtis \& D'Esposito, 2003; Miller \& Cohen, 2001; Sreenivasan, Curtis, \& D'Esposito, 2014), not VWM representations themselves.

However, the sensory recruitment model has since been questioned on at least two fronts. First, on logical grounds, if the same cortical machinery supports perception and memory, how can VWM be maintained in the face of ongoing perception? A potential answer is that the identical neurons within a region might not support both perception and working memory of a visual item: VWM representations are held globally within a sensory region, rather than being restricted to the specific subset of cortex that initially encoded the sensory input (Ester, Serences, \& Awh, 2009). To the degree that sensory recruitment is either global within sensory cortical regions or merely more flexible than requiring sustained activity of the very same neurons that initially encoded a stimulus, it may allow for some - though not unlimited - simultaneous perception and working memory. More recent research has shown that VWM representations in early sensory cortex may be disrupted (Bettencourt \& Xu, 2016) or biased (Lorenc, Sreenivasan, Nee, Vandenbroucke, \& D'Esposito, 2018) by ongoing visual input during the working memory delay, and only the parietal cortex clearly supports VWM representation under such more ecologically valid circumstances. Another neuroimaging study showed that disruption of early visual cortex representations negatively impacts behavior, suggesting that even if parietal representations play a role in VWM, they do not wholly explain it (Rademaker, Chunharas, \& Serences, 2019). Finally, functional neuroimaging techniques can extract the contents of VWM from signals in the prefrontal cortex (Ester, Sprague, \& Serences, 2015), although it is not clear if these signals are sufficient for or causally related to VWM. Thus, it remains unclear to what degree sensory recruitment is necessary or sufficient for VWM.

Another line of evidence questioning the sensory recruitment model comes from behavioral studies examining the kinds of errors made in reporting features of items maintained in VWM. Tamber-Rosenau, Fintzi, and Marois (2015) found that spatial crowding of a reported target item by flanking "crowder" stimuli led to similar changes in error when reporting item orientation for perceptual items and VWM items. However, they observed that participants made qualitatively distinct errors when reporting from perception and VWM: crowding in VWM led to an increase in outright substitution of one memory item for another but did not increase the pooling of multiple items that was evident alongside substitutions in purely perceptual crowding. They interpreted these results as evidence against a strong form of the sensory recruitment model (in which working memory depends on persistence of the original sensory activity across a delay) because the change in the nature of errors from perception to VWM suggests that either the two representations rely on distinct cortical machinery, or at least that there is a substantial change within the representation associated with the transition from perception to VWM. However, this purely behavioral research could not evaluate whether VWM might depend upon global or flexible representations in early sensory cortex (c.f., Ester et al., 2009), i.e., distinct neural populations from sensory representations within the same visual cortical brain regions. Such remapped representations would be consistent with a more flexible form of the sensory recruitment model in which VWM representations are maintained in visual cortex but do not necessarily require the very same neurons that initially represented the sensory stimulus before it was consolidated into VWM.

Recently, Harrison and Bays (2018, their Experiment 1) performed further behavioral studies that directly tested a key tenet of the strong sensory recruitment model (i.e., VWM representations as persistent activity in the same neurons that initially encoded the corresponding sensory inputs). By keeping the visual field eccentricity of a to-beremembered target item constant $\left(10^{\circ}\right.$ right of fixation along the horizontal meridian) while varying whether crowders were arranged vertically (tangentially) or horizontally (radially) with respect to the target, they elegantly manipulated the cortical distance between the target and inducers while keeping the real-world spatial distance identical (Fig. 1). To accomplish this, they cleverly took advantage of the known transformation from real-world spatial location to retinotopic cortical location (Holmes, 1918; Horton \& Hoyt, 1991; Inouye, 1909; Qiu et al., 2006), which has been demonstrated to modulate perceptual crowding (Toet \& Levi, 1992). Moreover, in contrast to Tamber-Rosenau et al. (2015), they ensured that any crowding effects they measured would derive purely from VWM by presenting the elements of the VWM sample sequentially. Critically, Harrison and Bays (2018) observed equivalent errors for reporting target orientation after a working memory delay regardless of whether the targets were crowded tangentially or radially. They interpreted this result as rejecting the sensory recruitment model because it predicts greater error with decreased cortical distance between the target and crowding inducers. 


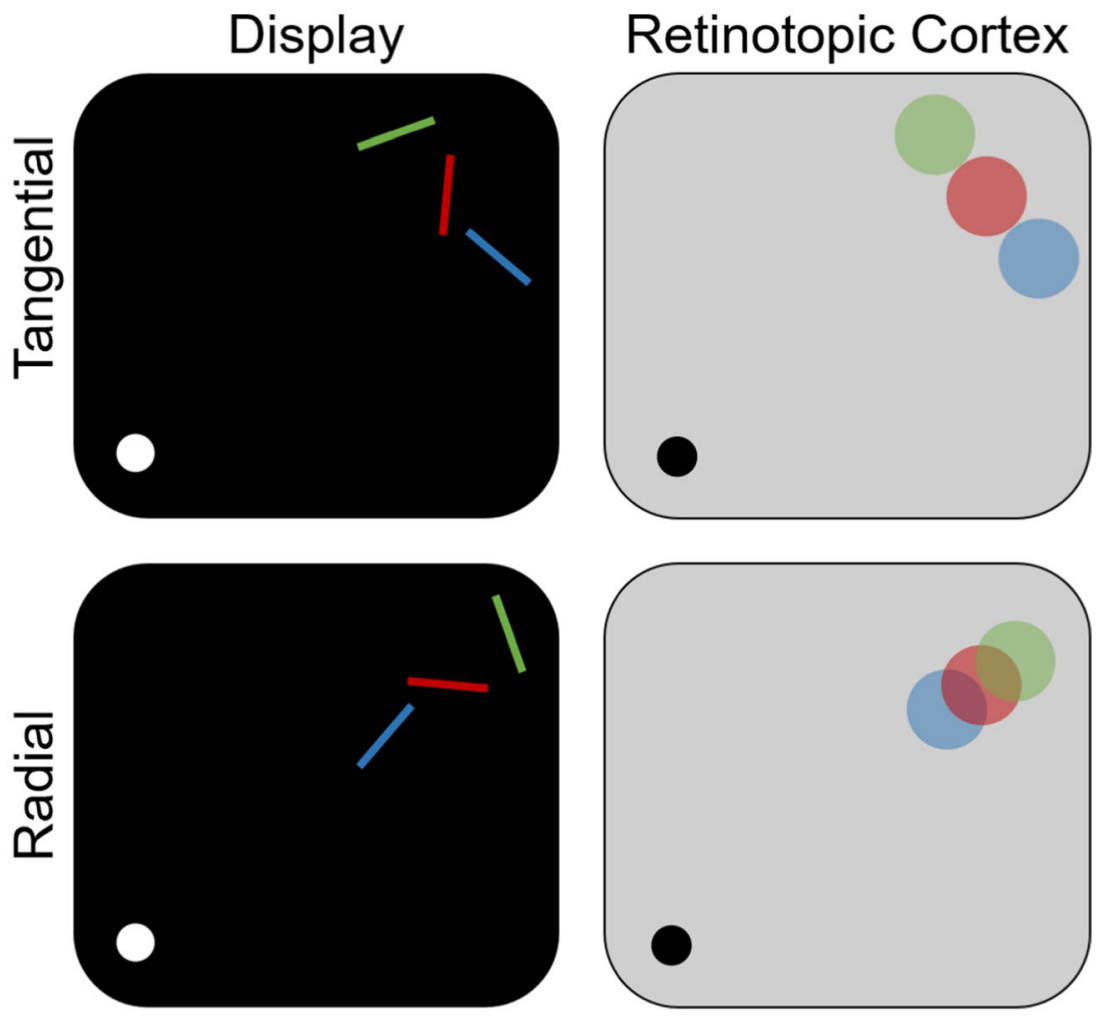

Fig. 1 Stimuli were presented in arrays centered on an imaginary circle of $10^{\circ}$ radius. Flanking stimuli (left) were presented either tangentially (top) or radially (bottom) with respect to the imaginary circle. Due to the configuration of visual cortex (right), radial (bottom) representations experience greater overlap in visual cortex than tangential representations (top). Note that visual stimuli are depicted as bars, and

The present study investigates a possible rescue for the sensory recruitment model, at least in its more flexible form (c.f., Ester et al., 2009). ${ }^{1}$ Ester et al. (2009)'s result suggests that participants could store memoranda globally throughout a sensory region. This is also consistent with a concern addressed by Tamber-Rosenau et al. (2015): that participants might flexibly remap visual memoranda from their original spatial locations (and corresponding retinotopically mapped cortical locations) to more spatially discrete cortical locations (Fig. 2) in order to strategically combat the effects of crowding. Reinforcing this concern, Pratte and Tong (2014) demonstrated that the lateralization of visual cortical representations of VWM information is mediated by task demand to maintain location- (hemifield-)specific information. In order to

\footnotetext{
${ }^{1}$ The finding that distraction can interfere with early sensory cortex representations of memoranda (Bettencourt \& Xu, 2016) rejects only the strong form of sensory recruitment because that study used distractors that subtended much of the central visual field, potentially precluding the remapping evident in Ester et al. (2009). However, it does not necessarily invalidate the more flexible form of the sensory recruitment hypothesis, in which early sensory brain regions play a critical role in VWM representation. This flexible version of sensory recruitment is consistent with the results of both Ester et al. (2009) and Tamber-Rosenau et al. (2015).
}

cortical representations are depicted as circles representing the full range of random orientations. Stimulus depictions are cartoons and are not to scale. All frames depict a single quadrant of a larger display (or the corresponding cortical representation). Figure adapted from Harrison and Bays (2018)

mitigate strategic remapping of stimulus representations, Tamber-Rosenau et al. (2015) required participants to report the exact location of the target rather than its orientation on half of their trials (with subjects only being cued on which feature to report - location or orientation - after the working memory delay). In other words, TamberRosenau et al. (2015) used a partial-report paradigm with respect to both item and feature to be reported. Without such a constraint on remapping, it is plausible that the observation of equal error regardless of crowder arrangement (Harrison \& Bays, 2018) might be driven by flexible remapping of initial perceptual representations (reliant on the cortical spacing of stimulus presentation locations) to novel VWM representations (with cortical spacing increased beyond the critical distance for crowding regardless of the initial item locations), or by reliance on less spatially precise representations in the parietal cortex (c.f., Bettencourt \& Xu, 2016).

To test our alternative account of Harrison and Bays (2018)'s result, we used a modified version of their task in which participants were required to maintain both orientation and precise location information for each VWM item - increasing the chances that cortical 


\section{Cortex}
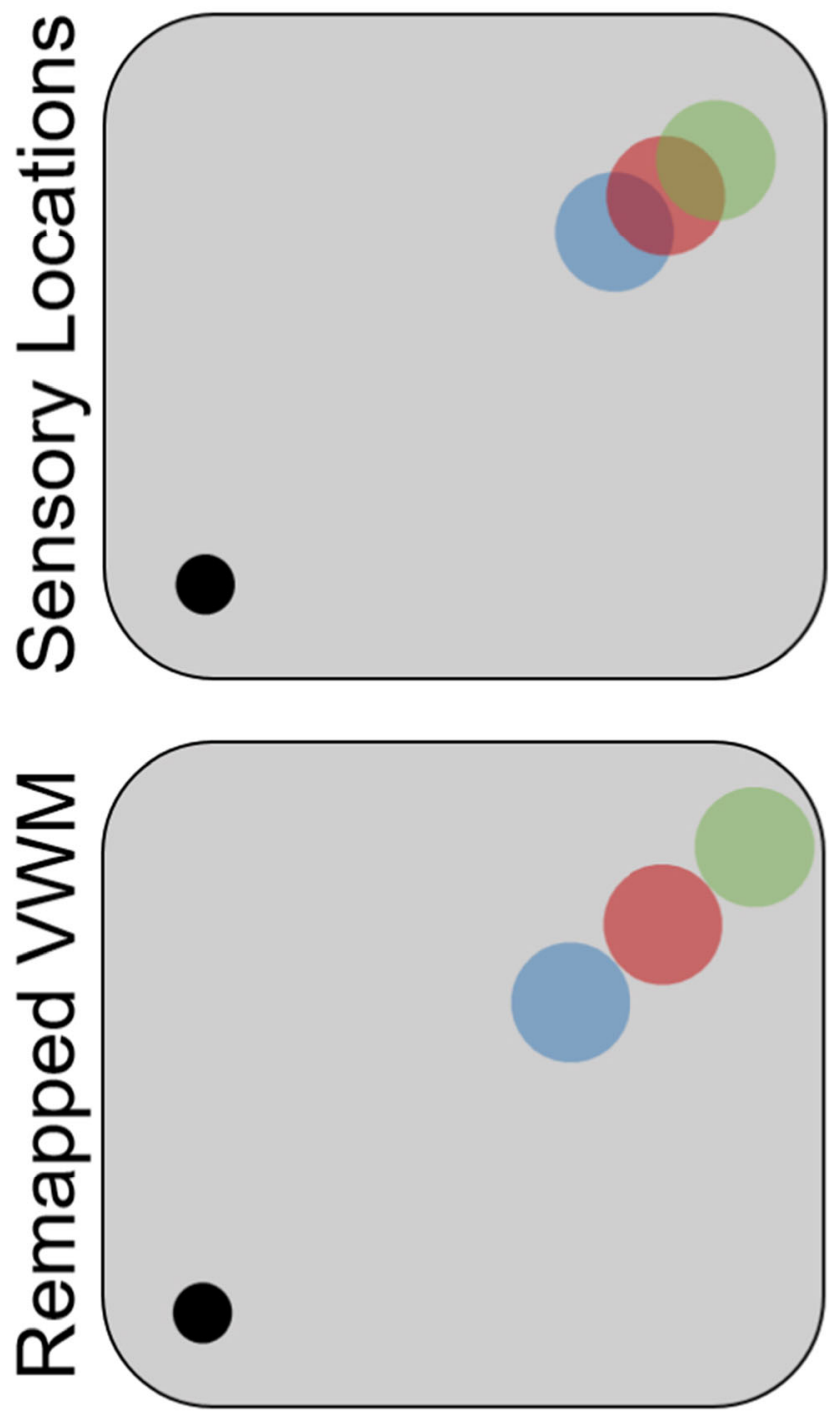

Fig. 2 It is plausible that cortical locations of sensory representations (top) may be remapped to new cortical locations in the same brain region during visual working memory (bottom) in order to mitigate crowding. Such an account is particularly plausible for sequential presentations that never experience perceptual crowding. In the present research, we included trials requiring precise location reports in order to discourage participants from using this remapping strategy

spacing of the VWM representations was successfully manipulated. Specifically, we combined the remappingconstraint strategy of Tamber-Rosenau et al. (2015) with the elegant radial/tangential crowder configuration strategy and sequential presentation approach of Harrison and Bays (2018). Built in to our approach is a replication of Harrison and Bays (2018) with greater sensitivity; this is important because their rejection of the sensory recruitment model depended on observing equivalent error for stimulus configurations intended to evoke distinct cortical distances, and their primary analysis found only moderate evidence for this invariance (Bayes factors of 2.97-4.21). ${ }^{2}$ On the other hand, should radial versus tangential crowder configuration (and thus, small vs. large cortical spacing) affect VWM reports, it would suggest that the more flexible form of sensory recruitment remains a plausible explanation for VWM.

\section{Method}

\section{Participants}

We recruited 25 adults from the University of Houston community under a protocol approved by the University of Houston Institutional Review Board. One participant was excluded because that participant's responses included such great error that the analysis models could not be fit. This led to a final sample of 24 participants (18 females, mean age 21.58 years, range $18-33$ ) in the main experiment. We recruited an additional 11 participants (eight females, mean age 19.18 years, range 18-21) for the control experiment. Participants were compensated via extra credit in their coursework and/or gift cards to Amazon.com.

\section{Power analysis}

We calculated that the study on which we based our task (Harrison \& Bays, 2018, their Experiment 1) should have had $90 \%$ power to detect effects as small as Cohen's $d=1.15$. Because our goal was to increase sensitivity, we targeted an effect of $\mathrm{d}=0.8$, which suggested a sample size of 19 (G*Power software version 3.1.9.2; Faul, Erdfelder, Lang, \& Buchner, 2007). However, it is possible that the increased task complexity of our design could inflate within-condition variance for each subject, leading to a small decrease in power not captured by traditional power analysis. ${ }^{3}$ Based on simulation results (not otherwise reported), a fivefold increase in withinparticipant, within-condition standard deviation can be compensated for by increasing sample size from 19 to 24 . Considering only a traditional power analysis, a sample of 24 was expected to have $90 \%$ power to detect an effect of size $\mathrm{d}=0.7$.

\footnotetext{
${ }^{2}$ Harrison and Bays (2018) also reported a combined Bayes factor of 12.5, obtained by multiplying the Bayes factors from their two experiments. However, this method of combining evidence may be erroneous (Rouder \& Morey, 2011). Using instead the meta-analytic method of Rouder and Morey (2011) as implemented in the R package BayesFactor version 0.9.12-4.2 (Morey, 2018), the combined Bayes factor should be 4.84, not 12.5. While such a result still supports the equivalence of errors across conditions, the evidence is much less substantial than would be suggested by a Bayes factor of 12.5 .

${ }^{3}$ We thank an anonymous reviewer for pointing out this possibility during review of the initial study protocol.
} 


\section{Comparison to Harrison and Bays (2018)}

We replicated Experiment 1 from Harrison and Bays (2018) with several extensions: First, we added trials requiring the report of target location (50\%) instead of orientation by using an extended partial-report paradigm. This forced participants to encode both the location and orientation features of each item on each trial, precluding them from maintaining only orientation in VWM. This partial-report paradigm increases the validity of comparing radial versus tangential orientation reports as a measure of their representations in VWM. Second, the present study doubled the overall number of trials, keeping the number of orientationreport trials identical to that in Harrison and Bays (2018). Third, we divided the experiment into three sessions due to the greater number of trials and the need for additional preparation of participants due to the increased task complexity. Fourth, we randomized stimulus array locations on a $10^{\circ}$ imaginary circle (instead of keeping all stimulus arrays directly to the right of fixation) so that target locations varied across trials, requiring the maintenance of exact spatial locations in VWM rather than categorical knowledge of a few frequently used locations.

\section{Procedure}

Participants sat at a desktop computer running KUbuntu Linux 16.04 LTS (https://kubuntu.org/) with a 21-in. CRT (actual viewing area: $40 \mathrm{~cm} \times 30 \mathrm{~cm}$ ) display at a resolution of $1,600 \times 1,200$ and a viewing distance of approximately $60 \mathrm{~cm}$ such that a pixel on the display corresponded to a visual angle of approximately $0.023^{\circ}$ (i.e., 43.4 pixels per degree of visual angle). Fixation was monitored by an Eyelink 1000 Plus eyetracker (SR Research, Ottawa, Ontario, Canada) configured for remote tracking $(500-\mathrm{Hz}$ sampling rate) without head fixation in order to increase participant comfort. Participants viewed stimuli on the monitor, heard auditory cues at an individually chosen comfortable volume, and responded using a standard mouse. The experiment was programmed using custom MATLAB (The Mathworks, Natick, MA, USA) code based upon that of Harrison and Bays (2018) ${ }^{4}$ and utilizing the Psychophysics Toolbox (Kleiner, Brainard, \& Pelli, 2007) and Eyelink Toolbox (Cornelissen, Peters, \& Palmer, 2002). The eyetracker was calibrated using standard procedures at the start of each session. Participants returned for three sessions each in order to complete the experiment; the first session lasted $90 \mathrm{~min}$ and subsequent sessions lasted $60 \mathrm{~min}$.

Participants began each trial by fixating a central fixation point for $500 \mathrm{~ms}$, which triggered a further delay of 250-750 $\mathrm{ms}$ (randomized, uniform distribution). Participants next viewed

\footnotetext{
${ }^{4}$ We thank William J. Harrison for generously sharing his MATLAB code and allowing its use and modification (William J. Harrison, personal communication, 18 July 2018).
}

a sequentially presented array of three bars $\left(2^{\circ} \times 0.2^{\circ}\right)$ with a $2^{\circ}$ center-to-center separation. Arrays (Fig. 1, left) were presented such that the central item was at a random location on an imaginary circle of radius $10^{\circ}$. On half of trials, the flanking items were presented along the meridian connecting the central item of the array to central fixation (i.e., radially; Fig. 1, bottom left). On the other half of trials, the flanking items were presented along a tangent intersecting the imaginary circle at the central array item location (Fig. 1, top left). The temporal order of presentation was random. Each item was colored red, green, or blue with the colors randomly distributed without replacement among the three stimuli on each trial. Each bar was presented for $500 \mathrm{~ms}$, and a 500-ms fixation-only screen was displayed between bars. After a further 500-ms working memory delay, participants viewed a colored item report cue (colored circle, $2^{\circ}$ diameter, central location), and heard an auditory feature report cue that consisted of a recorded voice speaking either of the words "tilt" or "place," cuing report of the orientation or exact location of the item matching the item report cue color.

On orientation-report trials, participants moved the mouse to adjust the orientation of a color-matched centrally presented orientation-report bar (which appeared simultaneously with the item report cue). The orientation-report bar initially had a random orientation. On location-report trials, participants moved the mouse to identify the location of the item whose color matched the probe circle. Upon mouse movement, the probe circle disappeared and a mouse cursor (circle, $1^{\circ}$ diameter, color matching the cued item) appeared at central fixation. On both kinds of trial, participants clicked the left mouse button to enter their responses after moving the mouse. ${ }^{5}$ For location trials, error was defined as the diagonal distance from the center of the cued bar to the clicked location. For orientation trials, error was defined as the angular difference between the reported and actual orientation of the cued item (Fig. 3).

All 36 trial types (two flanker configurations - radial, tangential; three array item locations that could be cued for report; three temporal positions for the cued item; two report features) were presented with six repetitions per session, or 18 repetitions total, for a total of 648 trials. Of the 36 trial types, we largely analyzed reports from the two flanker configurations separately, and the main analyses focused on trials in which the spatially central item was cued for orientation report. ${ }^{6}$ Following the

\footnotetext{
${ }^{5}$ In the initial study protocol, we planned to present trial-by-trial feedback. However, we ultimately elected not to present such feedback because of time constraints and because no such feedback was presented by Harrison and Bays (2018).

${ }^{6}$ Though it is possible to model errors in some location report tasks, the present paradigm was not optimized for such a study for two reasons: First, because of the constant target-flanker distances and restriction of target-flanker separation to two (radial, tangential) directions, location responses could be contaminated by long-term knowledge about stimulus array configurations. Second, our analyses would need major extension to accommodate directional error in a two-dimensional space that is subject to diminished acuity with eccentricity and to cortical magnification. Thus, we use the location task primarily to incentivize a representational strategy, not to assess results.
} 


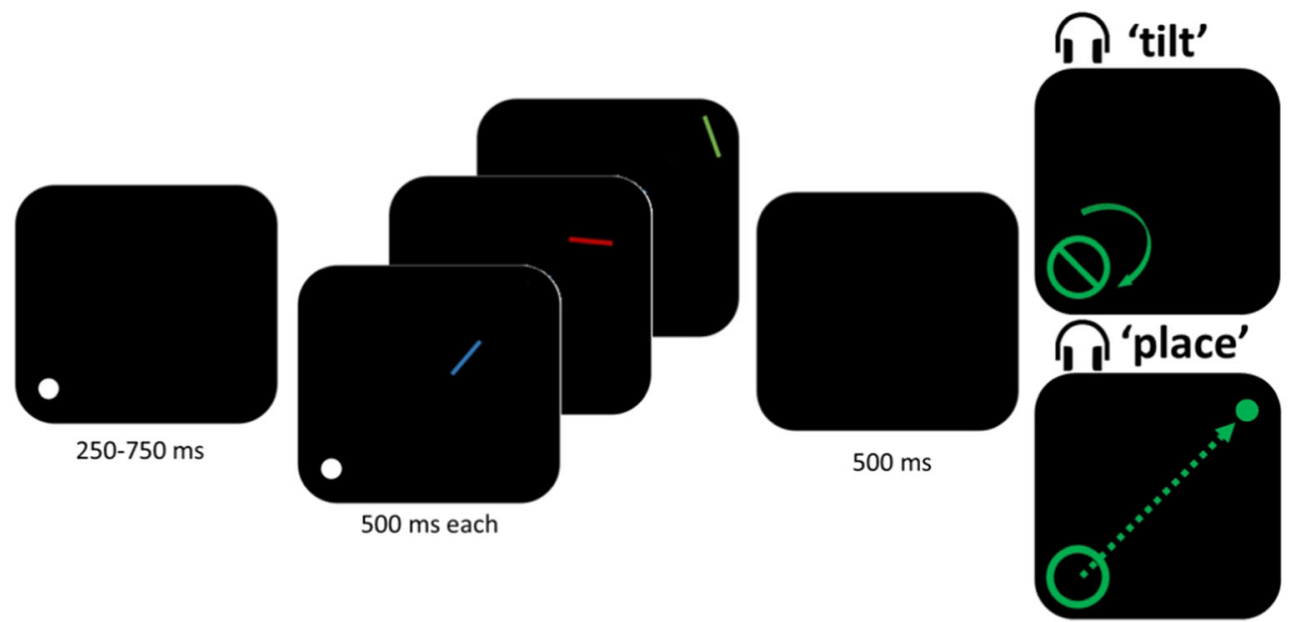

Fig. 3 A cartoon of the main experiment trial sequence - stimuli not presented to scale. Participants fixated on the central fixation point. Each bar was presented for $500 \mathrm{~ms}$, with 500-ms fixation-only screens between the bars. After a further 500-ms working memory delay from the offset of the final bar, participants viewed the colored report cue indicating which sample item was the target to be reported. Simultaneously with

procedure of Harrison and Bays (2018), we pooled trials across colors and presentation orders, and we pooled trials across sessions, leading to 54 center-item-cued radial orientationresponse trials and 54 corresponding tangential orientationresponse trials per participant.

We monitored fixation throughout each trial. When participants deviated from fixation by more than $2^{\circ}$ during the interval from the presentation of the initial stimulus through the entry of the report, ${ }^{7}$ the trial was interrupted and replaced after the presentation of a warning message.

During the first session only, participants completed practice trials in order to learn the task. Orientation- and locationreport tasks were practiced separately in mini-blocks of six trials (three radial arrays, three tangential arrays, other variables randomly selected per trial). Participants completed as many mini-blocks of each task as desired until they reported that they understood the task requirements and were consistently fixating as instructed.

\section{Control experiment}

It is possible that the simple change of presenting stimulus arrays at varying locations instead of in a consistent location from trial to trial could have driven differences between our results and those of Harrison and Bays (2018) - irrespective of the inclusion of location-report trials. ${ }^{8}$ Thus, we conducted an additional experiment to aid in interpretation of the results.

\footnotetext{
${ }^{7}$ Harrison and Bays (2018) only restricted eye movements until the onset of the report period, rather than its end. However, we also restricted eye movements during the report period (Tamber-Rosenau et al., 2015) so as to avoid any potential on-the-fly remapping of representations in visual cortex.

${ }^{8}$ We thank an anonymous reviewer for pointing out this possibility during review of the initial study protocol.
}

the color cue to the target item, participants heard the auditory cue indicating the feature to report. They then reported either the orientation or location of the target item using the mouse. The control experiment used an identical trial sequence (including presentation of the auditory "tilt" cue), except that no "place" trials were presented.

Specifically, 11 additional participants were recruited to approximately match the ten-participant sample size of Harrison and Bays (2018). As in our main experiment, we used variable array locations, but participants were only ever asked to report orientation, halving the total trial count. We thus conducted this control experiment in a single session. All other details were as in the main experiment.

\section{Results}

We tested whether VWM representations are influenced by the crowding anisotropy known from online visual perception (Toet \& Levi, 1992) and thought to stem from the retinotopic
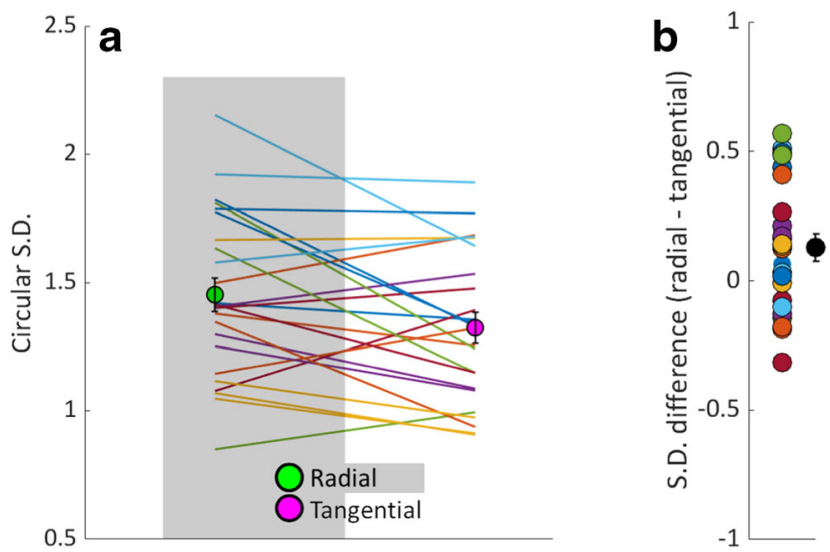

Fig. 4 Comparison of circular S.D. means across radial and tangential conditions in the main experiment. Error bars indicate \pm 1 SE. (A) Performance in each condition. Colored lines represent individual participants. Dots represent group means. (B) Difference between radial and tangential conditions. Colored circles indicate difference for each participant, black circle indicates the mean difference score 
configuration of the visual cortex. Specifically, we tested if radial configurations increased crowding compared to tangential configurations. We report the circular standard deviation of the orientation-report errors from the true orientation of the target item (Fig. 4) and compare these errors across crowder orientations via both standard t-tests and a Bayesian pairedsample t-test (Rouder, Speckman, Sun, Morey, \& Iverson, 2009). For the central item, radially configured arrays yielded higher orientation report error $(\mathrm{M}=1.45 \mathrm{rad}, \mathrm{SE}=0.07)$ compared to tangentially configured memoranda ( $\mathrm{M}=1.32$ $\mathrm{rad}, \mathrm{SE}=0.06$ ): Bayes factor (in favor of a difference $)=5.31$, $\mathrm{t}(23)=2.49, \mathrm{p}=.01$, one-tailed.

A secondary analysis, also exactly as conducted by Harrison and Bays (2018), used maximum likelihood estimation to fit the model of Bays, Catalao, and Husain (2009) to the orientation report errors. This model estimates the frequencies of trials on which participants report the cued item, erroneously report one of the flankers, or make a guess that is not informed by the stimulus array. The model also estimates non-guess report precision. The model was fit separately for each participant in two variants, either allowing each parameter to vary separately for radial and tangential trials or using the two trial types to fit a common set of parameters. The model fits were compared using Akaike Information Criterion (AIC) to determine whether a single set of parameters (suggesting equal crowding for radial and tangential arrays) or distinct sets of parameters (suggesting differential crowding for radial and tangential arrays) better account for the data.

The simpler three-parameter model (Fig. 5A), which assumed no difference between radial and tangential configurations, was a marginally better fit to the data than the more complex six-parameter model (Fig. 5B), which assumed distinct sets of parameters for radial and tangential trials: Summed $\Delta \mathrm{AIC}=63.90$ (simple model preferred in 23 out of 24 participants). This simple model yielded parameter

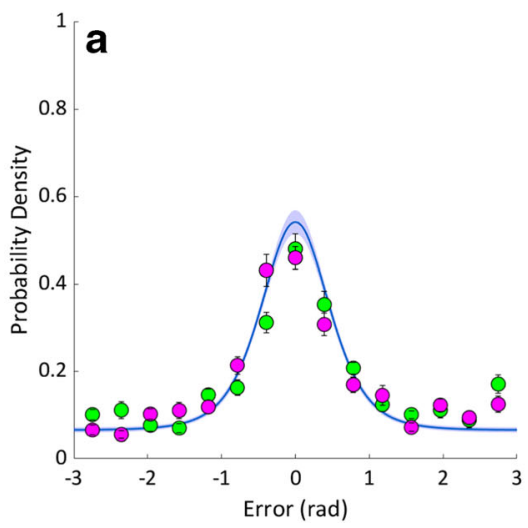

Fig. 5 Model fits in the main experiment. As in Harrison and Bays (2018), dots represent performance in equally spaced bins and lines represent the model. Error bars/bands represent \pm 1 SE. (A) The simpler three-parameter model, which assumed no influence of crowding estimates for precision $(5.07(0.13))$, swap rate $(0.14(0.00))$ and guess rate $(0.40(0.01))$. However, the mean difference of AIC values between the two models was low (mean $\Delta$ AIC $=$ 2.66), suggesting that the difference between the models was weak (see Burnham \& Anderson, 1998, p. 70; Lewandowsky \& Farrell, 2010, p. 185). We also compared $\mathrm{G}^{2} \log$-likelihood ratios in each participant to evaluate if the models were meaningfully different in each participant. For 23 of the 24 participants, the models were not significantly different (in these 23 participants: mean $\Delta \mathrm{G}^{2}=2.84$, $\min \Delta \mathrm{G}^{2}=.70$, $\max \Delta \mathrm{G}^{2}=$ 5.46 , all ps $>.14$ ), and for the remaining participant the sixparameter model was preferred $\left(\Delta G^{2}=14.74, p=0.002\right)$.

We also examined the log-likelihoods to determine if either model was actually a good fit to the observed data, separately for each participant. Both models were significantly discrepant from the observed data for each subject (simple threeparameter model: mean $\mathrm{G}^{2}=340$, $\min \mathrm{G}^{2}=271$, $\max \mathrm{G}^{2}=$ 384 , all ps $<10^{-57}$; complex six-parameter model: mean $\mathrm{G}^{2}=$ 336, $\operatorname{min~}^{2}=269, \max \mathrm{G}^{2}=383$, all $\mathrm{ps}<10^{-54}$ ). Therefore, neither model provided a particularly good description of the orientation report errors.

We also evaluated the utility of the modeling using a less formal approach: we visually inspected the per-participant parameter estimates in order to look for unreasonable patterns of trade-offs between parameters. Specifically, when we observed the individual swap and guess rates, there seemed to be a trade-off between those two parameters (see Supplemental Fig. 1). Supporting that evaluation, we observed significant negative correlations between swap and guess rates in the simpler model $(\mathrm{r}=-.57, \mathrm{p}<.005)$ and for both radial $(\mathrm{r}=-.66, \mathrm{p}<.001)$ and tangential $(\mathrm{r}=-.43, \mathrm{p}<.05)$ configurations in the more complex model. This suggests that the models have difficulty distinguishing between guesses and swaps because there is little reason to expect a negative correlation between these parameters - instead, one would expect that both guesses and swaps would increase when a

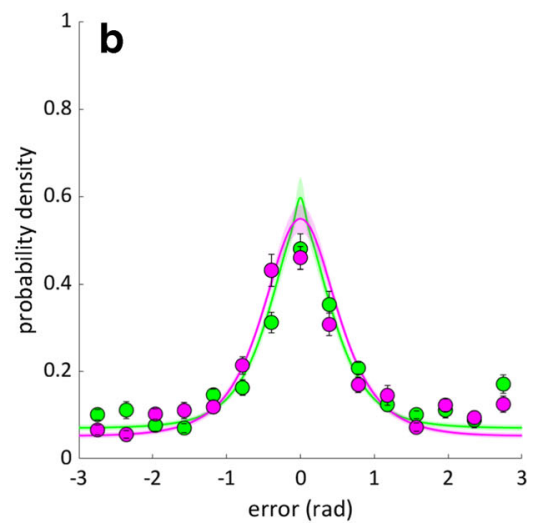

anisotropy across radial and tangential conditions. (B) The more complex six-parameter model, which assumed distinct sets of parameters for radial and tangential conditions 
participant found the task to be difficult. A failure to properly estimate any model parameter undermines all parameter estimates from the modeling approach because parameters are not estimated independently from one another.

Taking together the parameter trade-offs in the models, the weak differences in the model fits, and the discrepancies between model fits and data for each model, it is our view that the modeling results are of marginal value. Instead, we favor evaluating the circular standard deviation error results directly, as these make far fewer assumptions and remain dispositive as to the presence or absence of crowding. To reiterate, the direct examination of errors indicated a Bayes factor $>5$ in favor of the existence of a radial/tangential crowding anisotropy for sequentially presented visual memoranda.

In addition to our planned analyses, we also conducted a follow-up analysis of another measure of crowding. Specifically, for the three-element VWM arrays in any configuration, the center item is subject to more crowding than the flanking items because the center item is adjacent to two crowders but the flankers are adjacent to only one crowder. Thus, another way to test crowding is to compare orientationreport errors between center-item-report and flanker-itemreport trials. (Note that we use the terms flanker target and peripheral target interchangeably, as the flankers are on the
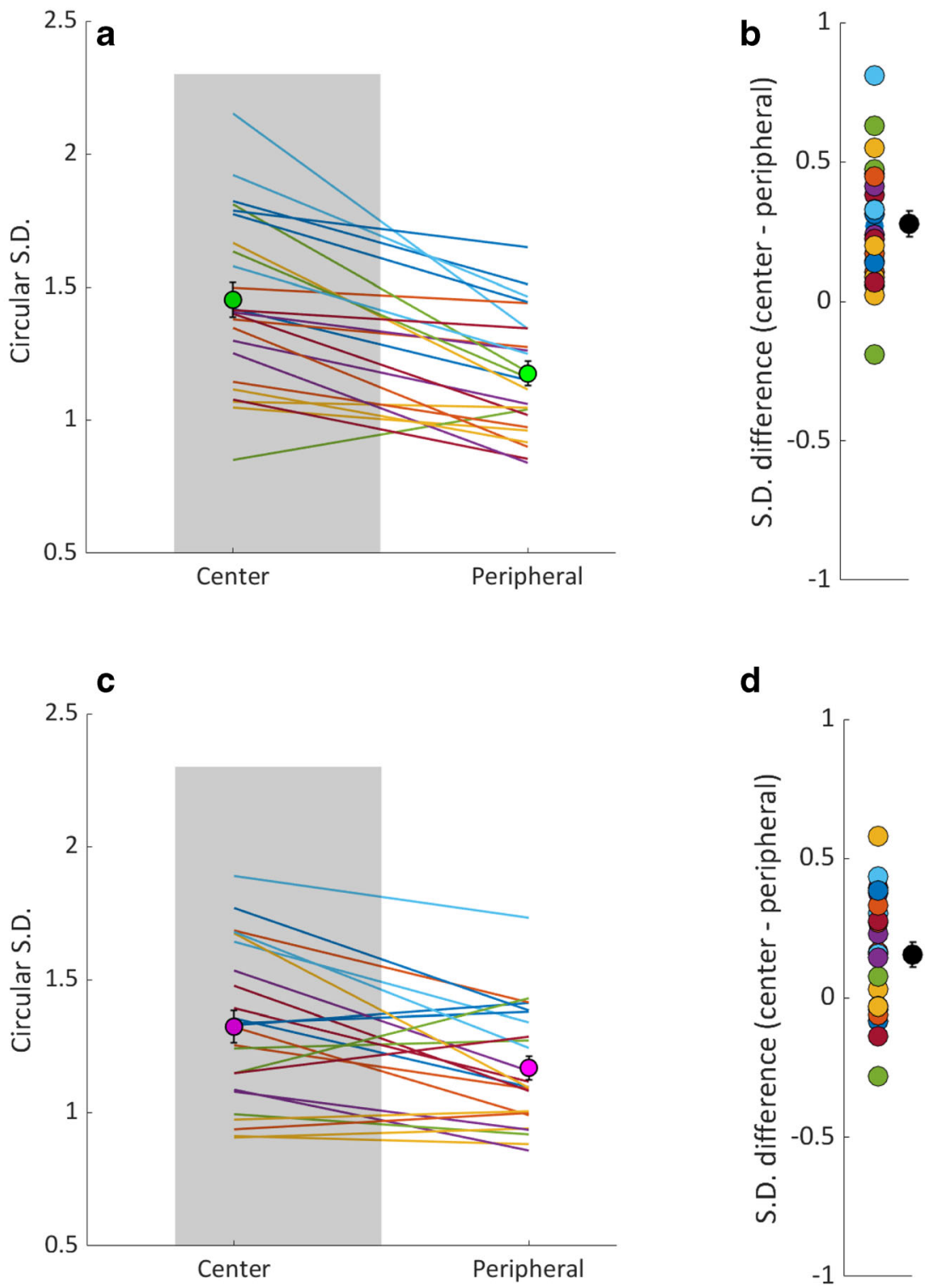

Fig. 6 Comparison of circular S.D. means across central and peripheral targets in the main experiment. Error bars indicate $\pm 1 \mathrm{SE}$. In panels A and $\mathrm{C}$, colored lines represent individual participants and circles represent group means. In panels B and D, colored circles represent individual participants and black circles represent group means. (A) Radial condition, in which eccentricity varies across central and peripheral targets, broken out by target location. (B) Difference across target locations in radial condition. (C) Tangential condition, in which eccentricity is equivalent across central and peripheral targets, broken out by target location. (D) Difference across target locations in tangential condition 
periphery of the array.) For radial configurations, we observed higher errors for center target $(\mathrm{M}=1.45 \mathrm{rad}, \mathrm{SE}=0.07)$ than flanker targets $(\mathrm{M}=1.18 \mathrm{rad}, \mathrm{SE}=0.05)$, Bayes factor (in favor of a difference $)=14316.04, \mathrm{t}(23)=6.17, \mathrm{p}<.001$, onetailed (Fig. 6A, B). However, the radial results are confounded with distance, as flankers were always either closer or further from fixation than central targets. On the other hand, the eccentricity of each VWM array item is nearly identical for tangential configurations. On tangential trials, we observed higher orientation report errors for central targets $(\mathrm{M}=1.32$ $\mathrm{rad}, \mathrm{SE}=0.06)$ compared to peripheral targets $(\mathrm{M}=1.17 \mathrm{rad}$, $\mathrm{SE}=0.05$ ), Bayes factor (in favor of a difference) $=41.86$, $\mathrm{t}(23)=3.53, \mathrm{p}<.001$, one-tailed (Fig. 6C, D). Though it was not planned, this analysis provides further strong evidence for crowding within VWM representations. We also applied this analysis to the data of Harrison and Bays (2018) and observed some weak evidence for crowding in tangential displays, but the errors did not reflect the strong crowding effect observed in our data (radial condition: no significant difference between center $(\mathrm{M}=0.86 \mathrm{rad}, \mathrm{SE}=0.09)$ and peripheral targets $(\mathrm{M}=$ $0.83 \mathrm{rad}, \mathrm{SE}=0.09$ ), Bayes factor (in favor of a difference) $=$ $1.14, \mathrm{t}(9)=1.38, \mathrm{p}=.10$, one-tailed; tangential condition: higher errors for center $(\mathrm{M}=0.88 \mathrm{rad}, \mathrm{SE}=0.09)$ target than peripheral targets $(\mathrm{M}=0.81 \mathrm{rad}, \mathrm{SE}=0.09$ ), Bayes factor (in favor of a difference) $=3.76, t(9)=2.34, p=.02$, one-tailed). The discrepancy between our results and those of Harrison and Bays (2018) further suggests that in their study, the absence of location trials led participants to adopt strategies such as remapping to minimize crowding.

Finally, we leveraged the location-report data for a single analysis designed to assess whether potential individual differences in flexible remapping of VWM representations could explain differences in VWM crowding across individuals. Specifically, we reasoned that participants who were more prone to engage in remapping would make greater location errors but experience less VWM crowding, compared to participants who were less prone to remapping. Thus, we computed the mean location-report error magnitude (without respect to direction) for all trials in which the central item was cued (i.e., location errors without distinguishing radial and tangential arrays). Across participants, there was not a significant association between these location report errors and the amount of crowding (as indexed by the difference between radial and tangential orientation report errors; Pearson $\mathrm{r}=$ $-.18, \mathrm{p}=.40)$ (Fig. 7). Therefore, it is unlikely that individual differences in task strategies strongly drive VWM crowding effects.

\section{Control experiment results}

In the control experiment, participants did not need to encode location-bound VWM representations because only orientation was ever probed. In contrast to the main experiment, the

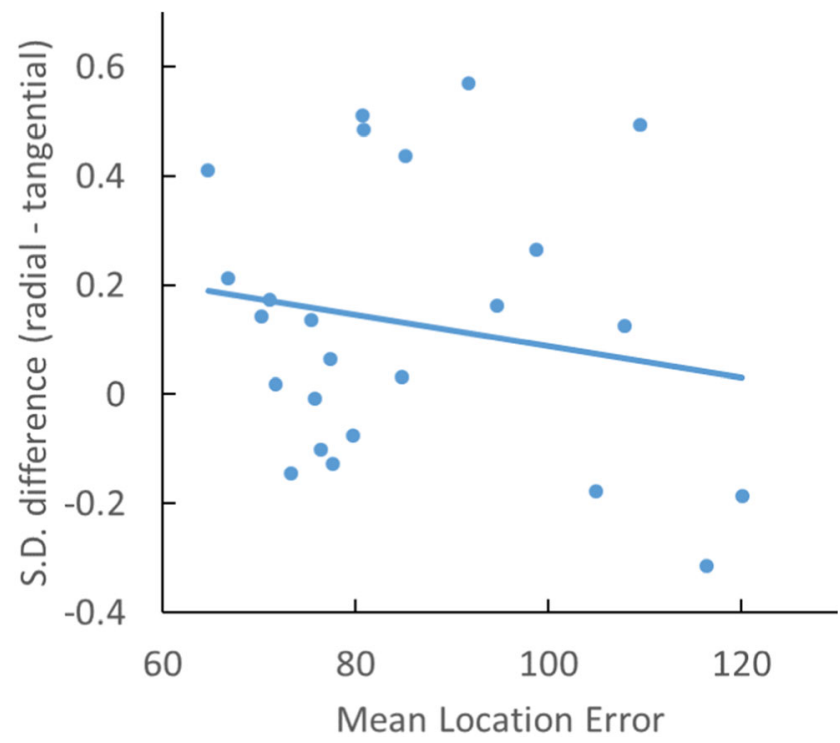

Fig. 7 Relationship between location and orientation errors in main experiment: mean absolute magnitude of location report errors (in pixels) across all trials versus mean difference between radial and tangential report errors for each participant

control experiment (Fig. 8) closely replicated the results of Experiment 1 of Harrison and Bays (2018): there was no difference between orientation report errors for the center target across radial $(\mathrm{M}=1.16 \mathrm{rad}, \mathrm{SE}=0.07)$ and tangential $(\mathrm{M}=$ $1.24 \mathrm{rad}, \mathrm{SE}=0.11$ ) configurations (Bayes factor (in favor of invariance $)=2.54, \mathrm{t}(10)=-0.81, \mathrm{p}=.78$, one-tailed). To follow up on this analysis, we also compared the orientation report errors across central and flanker targets for radial and tangential configurations. The results favored equivalence between central and flanker target errors (Fig. 9), both for radial (confounded with eccentricity) arrays (Bayes factor (in favor of invariance $)=1.46, t(10)=-1.46, p=.91$, one-tailed $)$, and tangential arrays (Bayes factor (in favor of invariance) $=2.80$, $\mathrm{t}(10)=0.66, \mathrm{p}=.26$, one-tailed) (Fig. 9). In addition, the comparison between simple three-parameter versus complex six-parameter models favored the simpler model (summed $\Delta$ AIC $=24.09 ; 10$ out of 11 participants; mean (SE) of parameter estimates: precision $=5.45(0.37), \mathrm{p}(\mathrm{swap})=0.13$ $(0.01), p($ guess $)=0.30(0.02))$ (Supplemental Fig. 2). Thus, supporting the view that representing location information drives participants to represent VWM items in early visual cortex, participants seem not to have represented VWM items retinotopically when location information was irrelevant to the task at hand.

\section{Discussion}

In the present research, we aimed to re-evaluate the sensory recruitment model of VWM by preventing a strategy by which participants could improve task performance by sacrificing 

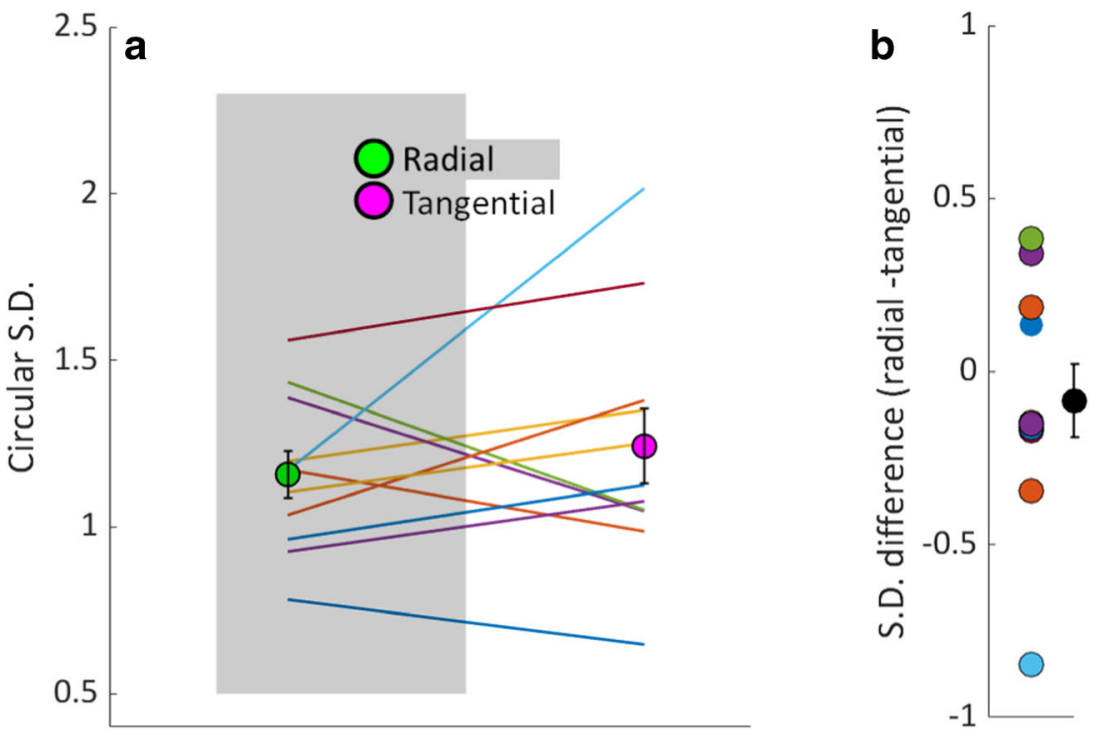

Fig. 8 Comparison of circular S.D. means across radial and tangential conditions in the control experiment (compare to Fig. 4, main experiment results). Error bars indicate \pm 1 SE. (A) Performance in each condition. Colored lines represent individual participants. Dots represent group means. (B) Difference between radial and tangential conditions. Colored circles indicate difference for each participant, black circle indicates the mean difference score

To be able to meaningfully require location reports, we presented the VWM sample arrays at varying locations. To eliminate the possibility that this distinct display geometry from that of Harrison and Bays (2018) is what drove our results, we conducted a control experiment in which we removed the location-report trials. When the need for featurelocation binding was removed, the results became consistent with those of Harrison and Bays (2018), confirming that it was location-binding, not display geometry, that drove our results in the main experiment. Therefore, our overall results support the view that the sensory recruitment model, especially in its flexible form, can explain the most typically studied locationbound forms of VWM.

The crowding anisotropy we report here is likely an underestimate. This is because we, in order to replicate Harrison and Bays (2018) as closely as possible, used color-heterogeneous VWM arrays. Distinct target and flanker colors are known to decrease crowding in visual perception, while more similar colors lead to increased crowding (Kennedy \& Whitaker, 2010). Future studies seeking to compare VWM and perceptual crowding should use single-color arrays and cue target identities using other features such as presentation order (c.f., Ahmad et al., 2017).

Other recent research has evaluated the sensory recruitment model by examining whether VWM representations show additional hallmarks of early visual cortex (other than crowding) known from perceptual vision. For example, Bloem et al. (2018) investigated whether VWM representations exhibited contrast normalization. While they observed normalization for perceptual items, they did not find such an effect for VWM representations, leading them to argue against the sensory 

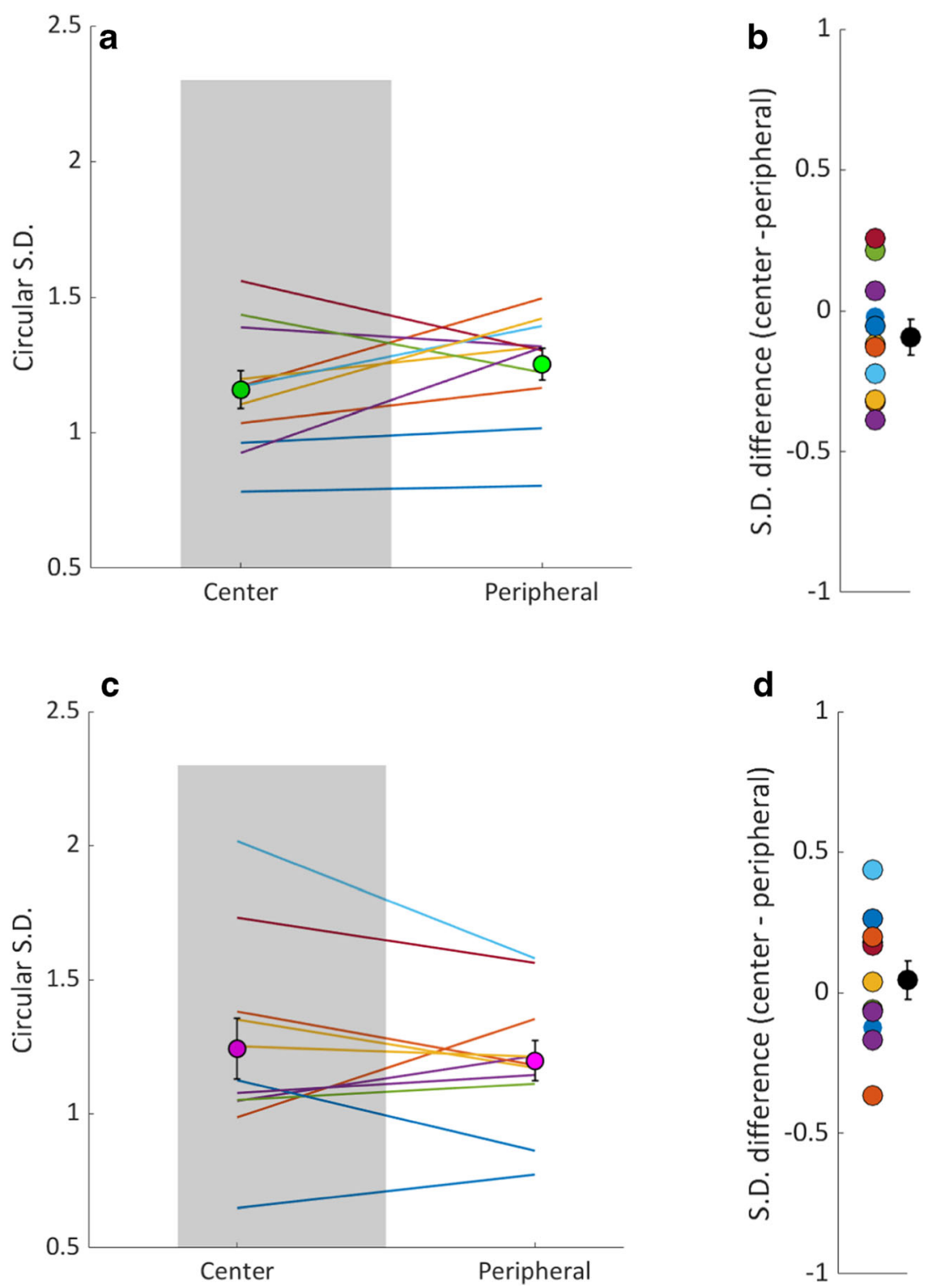

Fig. 9 Comparison of circular S.D. means across central and peripheral targets in the control experiment (compare to Fig. 6, main experiment results). Error bars indicate $\pm 1 \mathrm{SE}$. In panels $\mathrm{A}$ and $\mathrm{C}$, colored lines represent individual participants and circles represent group means. In panels $\mathrm{B}$ and $\mathrm{D}$, colored circles represent individual participants and black circles represent group means. (A) Radial condition, in which eccentricity

recruitment model. However, Bloem et al. (2018) relied on arrays of central and surround gratings, always positioned consistently, with no object-location bindings - similar to Harrison and Bays (2018). On the other hand, Fang, Ravizza, and Liu (2019) tested the sensory recruitment model using surround suppression. They presented their memoranda simultaneously and used location cues to indicate to-beremembered items, and they observed surround suppression in VWM representations. Considering these studies together, spatially specific encoding or maintenance seems crucial for interpreting if the VWM representations are sustained by activity in early visual cortex. However, we note that minimal varies across central and peripheral targets, broken out by target location. (B) Difference across target locations in radial condition. (C) Tangential condition, in which eccentricity is equivalent across central and peripheral targets, broken out by target location. (D) Difference across target locations in tangential condition

location requirements may not be sufficient to evoke reliance on early visual cortical representations: Our initial effort to gather data for the present study (Yoruk \& TamberRosenau, 2019) contained a technical error that led radial and tangential trials to be blocked in their presentation order, rather than randomized as desired. Blocking the radial/ tangential presentation order - even when half of trials required location report - could create the opportunity for participants to encode location only coarsely (e.g., near/middle/ far in a direction during radial blocks, or left/middle/right in a direction during tangential blocks), leading to reliance on remapping strategies or on representations outside the early 
visual cortex. Consistent with this supposition, we did not observe anisotropic crowding in those blocked data.

In the present study, we used a behavioral method to exert experimental control and constrain strategic remapping of VWM representations. Other approaches could serve to evaluate sensory recruitment. For instance, careful retinotopic mapping using neuroimaging could examine whether the initial cortical locations in which the target and crowders are represented are the only early sensory cortex locations in which they are represented at the end of the VWM delay (just prior to report), or whether visual cortex activations shift during the delay - consistent with remapping. While this approach would directly evaluate remapping, it has the significant disadvantage of requiring an extended delay period long enough to separate slow hemodynamic correlates of initial sensory neural activity from VWM representations. This difference could be important as it is not clear if the same representational mechanisms are in play over short and long durations: Some evidence favors distinct representational stores within VWM playing out over time (Pinto, Sligte, Shapiro, \& Lamme, 2013; Sligte, Scholte, \& Lamme, 2008, 2009; Sligte, Vandenbroucke, Scholte, \& Lamme, 2010; Sligte, Wokke, Tesselaar, Scholte, \& Lamme, 2011; van Moorselaar, Olivers, Theeuwes, Lamme, \& Sligte, 2015; Vandenbroucke, Sligte, de Vries, Cohen, \& Lamme, 2015; Vandenbroucke, Sligte, \& Lamme, 2011). However, other researchers have argued that multiple stages of VWM are not necessary to explain behavior (Makovski, 2012; Matsukura \& Hollingworth, 2011; also see, e.g., Pertzov, Manohar, \& Husain, 2017, for a discussion of how a single representational store can change in capacity over time and competition between memoranda) or that attentional fluctuations can explain changes in VWM representations over time (Jacob, Breitmeyer, \& Trevino, 2014). Furthermore, due to the coarse resolution of fMRI, it is not clear that neuroimaging could easily distinguish small remapping distances that would be sufficient to move a representation beyond the critical distance for crowding. Although our behavioral task cannot provide neuronal evidence for direct correspondence between spatial locations of stimuli and retinotopic maps in sensory cortex, it provides clear behavioral evidence that crowding effects exists for VWM representations when strategic remapping is restricted. Therefore, our findings endorse the sensory recruitment model, at least in its flexible form.

With the assurance from our results that VWM crowding exists and reflects the same radial/tangential anisotropy as visual crowding, the next step should be to quantify the extent of VWM crowding. Previous research has carefully quantified the relationship between eccentricity and target-flanker distance in evoking crowding (e.g., Bouma, 1970), as well as upper versus lower visual field (He, Cavanagh, \& Intriligator, 1996). Though they are beyond the scope of the present paper, there are a plethora of additional visual crowding properties (see reviews by Levi, 2008; Whitney \& Levi, 2011) whose persistence in or absence from VWM remain to be investigated.

In conclusion, VWM is flexible and it can rely on multiple possible representations (Bettencourt \& Xu, 2016; Ester et al., 2015; Rademaker et al., 2019; Serences, 2016). When VWM representations with high spatial precision are needed, as in classic change-detection paradigms and as expected of maximally "image-like" or "visual" VWM representations, they exhibit hallmarks of reliance on early visual cortex. By providing evidence for VWM crowding when spatial precision is required, our study supports the sensory recruitment model.

Author note We thank William J. Harrison for generously sharing his MATLAB code for stimulus presentation and data analysis; Bruno Breitmeyer, Don Foss, and Brandon Carlos for helpful discussions and critiques of an earlier version of this manuscript; and Yenifer Morales, Giang Le, Faith Huynh, and Ryan Farias for help with data collection.

Open Practices Statement The data for all experiments are available at https://osf.io/dv485/. This study was preregistered via submission in the Registered Report or Replication (RRR) format to Psychonomic Bulletin \& Review.

\section{References}

Ahmad, J., Swan, G., Bowman, H., Wyble, B., Nobre, A. C., Shapiro, K. L., \& McNab, F. (2017). Competitive interactions affect working memory performance for both simultaneous and sequential stimulus presentation. Scientific Reports, 7(1), 4785. https://doi.org/10.1038/ s41598-017-05011-x

Bays, P. M., Catalao, R. F., \& Husain, M. (2009). The precision of visual working memory is set by allocation of a shared resource. $J$ Vis, 9(10), 7 1-11. https://doi.org/10.1167/9.10.7

Bettencourt, K. C., \& Xu, Y. (2016). Decoding the content of visual short-term memory under distraction in occipital and parietal areas. Nature Neuroscience, 19(1), 150-157. https://doi.org/10.1038/nn. 4174

Bloem, I. M., Watanabe, Y. L., Kibbe, M. M., \& Ling, S. (2018). Visual Memories Bypass Normalization. Psychological Science, 29(5), 845-856. https://doi.org/10.1177/0956797617747091

Bouma, H. (1970). Interaction effects in parafoveal letter recognition. Nature, 226(5241), 177-178.

Burnham, K. P., \& Anderson, D. R. (1998). Model Selection and Multimodel Inference. Berlin: Springer.

Cornelissen, F. W., Peters, E. M., \& Palmer, J. (2002). The Eyelink Toolbox: eye tracking with MATLAB and the Psychophysics Toolbox. Behavior Research Methods, Instruments, \& Computers, 34(4), 613-617.

Courtney, S. M., Ungerleider, B. G., Keil, K., \& Haxby, J. V. (1997). Transient and sustained activity in a distributed neural system for human working memory. Nature, 386(6625), 608-611. 
Curtis, C. E., \& D'Esposito, M. (2003). Persistent activity in the prefrontal cortex during working memory. Trends in Cognitive Sciences, 7(9), 415-423.

Ester, E. F., Serences, J. T., \& Awh, E. (2009). Spatially global representations in human primary visual cortex during working memory maintenance. The Journal of Neuroscience, 29(48), 15258-15265. https://doi.org/10.1523/JNEUROSCI.4388-09.2009

Ester, E. F., Sprague, T. C., \& Serences, J. T. (2015). Parietal and Frontal Cortex Encode Stimulus-Specific Mnemonic Representations during Visual Working Memory. Neuron, 87(4), 893-905. https://doi. org/10.1016/j.neuron.2015.07.013

Fang, M. W. H., Ravizza, S. M., \& Liu, T. (2019). Attention induces surround suppression in visual working memory. Psychonomic Bulletin \& Review, 26(6), 1925-1932. https://doi.org/10.3758/ s13423-019-01624-7

Faul, F., Erdfelder, E., Lang, A. G., \& Buchner, A. (2007). G*Power 3: a flexible statistical power analysis program for the social, behavioral, and biomedical sciences. Behavior Research Methods, 39(2), 175191

Harrison, S. A., \& Tong, F. (2009). Decoding reveals the contents of visual working memory in early visual areas. Nature, 458(7238), 632-635.

Harrison, W. J., \& Bays, P. M. (2018). Visual Working Memory Is Independent of the Cortical Spacing Between Memoranda. The Journal of Neuroscience, 38(12), 3116-3123. https://doi.org/10. 1523/JNEUROSCI.2645-17.2017

He, S., Cavanagh, P., \& Intriligator, J. (1996). Attentional resolution and the locus of visual awareness. Nature, 383(6598), 334-337. https:// doi.org/10.1038/383334a0

Holmes, G. (1918). Disturbances of vision by cerebral lesions. British Journal of Ophthalmology, 2, C353-C384.

Horton, J. C., \& Hoyt, W. F. (1991). The representation of the visual field in human striate cortex. A revision of the classic Holmes map. Archives of Ophthalmology, 109(6), 816-824.

Inouye, T. (1909). Die Sehstorungen bei Schussverletzungen der kortikalen Sehsphare. Leipzig, Germany: W Engelmann.

Jacob, J., Breitmeyer, B., \& Trevino, M. (2014). The first four seconds: An assessment of post-stimulus processing in visual short-term memories. Journal of Vision, 14(10), 856.

Kennedy, G. J., \& Whitaker, D. (2010). The chromatic selectivity of visual crowding. Journal of Vision, 10(6), 15. https://doi.org/10. $1167 / 10.6 .15$

Kleiner, M., Brainard, D., \& Pelli, D. (2007). What's new in Psychtoolbox-3? Perception, 36, 14-14.

Kriegeskorte, N., Goebel, R., \& Bandettini, P. (2006). Information-based functional brain mapping. Proceedings of the National Academy of Sciences of the United States of America, 103(10), 3863-3868.

Levi, D. M. (2008). Crowding-an essential bottleneck for object recognition: a mini-review. Vision Research, 48(5), 635-654. https://doi. org/10.1016/j.visres.2007.12.009

Levy, R., \& Goldman-Rakic, P. S. (1999). Association of storage and processing functions in the dorsolateral prefrontal cortex of the nonhuman primate. The Journal of Neuroscience, 19(12), 5149-5158.

Levy, R., \& Goldman-Rakic, P. S. (2000). Segregation of working memory functions within the dorsolateral prefrontal cortex. Experimental Brain Research, 133(1), 23-32.

Lewandowsky, S., \& Farrell, S. (2010). Computational modeling in cognition: Principles and practice: SAGE Publications.

Lorenc, E. S., Sreenivasan, K. K., Nee, D. E., Vandenbroucke, A. R. E., \& D'Esposito, M. (2018). Flexible Coding of Visual Working Memory Representations during Distraction. The Journal of Neuroscience, 38(23), 5267-5276. https://doi.org/10.1523/ JNEUROSCI.3061-17.2018

Luck, S. J., \& Vogel, E. K. (1997). The capacity of visual working memory for features and conjunctions. Nature, 390(6657), 279281. https://doi.org/10.1038/36846
Ma, W. J., Husain, M., \& Bays, P. M. (2014). Changing concepts of working memory. Nature Neuroscience, 17(3), 347-356. https:// doi.org/10.1038/nn.3655

Makovski, T. (2012). Are multiple visual short-term memory storages necessary to explain the retro-cue effect? Psychonomic Bulletin \& Review, 19(3), 470-476. https://doi.org/10.3758/s13423-012-02359

Matsukura, M., \& Hollingworth, A. (2011). Does visual short-term memory have a high-capacity stage? Psychonomic Bulletin \& Review, 18(6), 1098-1104. https://doi.org/10.3758/s13423-011-0153-2

Miller, E. K., \& Cohen, J. D. (2001). An integrative theory of prefrontal cortex function. Annual Review of Neuroscience, 24, 167-202. https://doi.org/10.1146/annurev.neuro.24.1.167

Morey, R. D. (2018). BayesFactor: An R package for Bayesian data analysis. Retrieved from https://richarddmorey.github.io/ BayesFactor/

Pertzov, Y., Manohar, S., \& Husain, M. (2017). Rapid Forgetting Results From Competition Over Time Between Items in Visual Working Memory. Journal of Experimental Psychology-Learning Memory and Cognition, 43(4), 528-536. https://doi.org/10.1037/ $\mathrm{x} \operatorname{lm} 0000328$

Pinto, Y., Sligte, I. G., Shapiro, K. L., \& Lamme, V. A. (2013). Fragile visual short-term memory is an object-based and location-specific store. Psychonomic Bulletin \& Review, 20(4), 732-739. https://doi. org/10.3758/s13423-013-0393-4

Pratte, M. S., \& Tong, F. (2014). Spatial specificity of working memory representations in the early visual cortex. Journal of Vision, 14(3), 22. https://doi.org/10.1167/14.3.22

Qiu, A., Rosenau, B. J., Greenberg, A. S., Hurdal, M. K., Barta, P., Yantis, S., \& Miller, M. I. (2006). Estimating linear cortical magnification in human primary visual cortex via dynamic programming. Neuroimage, 31(1), 125-138. https://doi.org/10.1016/j.neuroimage. 2005.11.049

Rademaker, R. L., Chunharas, C., \& Serences, J. T. (2019). Coexisting representations of sensory and mnemonic information in human visual cortex. Nature Neuroscience https://doi.org/10.1038/ s41593-019-0428-x

Rouder, J. N., \& Morey, R. D. (2011). A Bayes factor meta-analysis of Bem's ESP claim. Psychonomic Bulletin \& Review, 18(4), 682-689. https://doi.org/10.3758/S13423-011-0088-7

Rouder, J. N., Speckman, P. L., Sun, D. C., Morey, R. D., \& Iverson, G. (2009). Bayesian $t$ tests for accepting and rejecting the null hypothesis. Psychonomic Bulletin \& Review, 16(2), 225-237. https://doi. org/10.3758/Pbr.16.2.225

Schneegans, S., \& Bays, P. M. (2017). Neural Architecture for Feature Binding in Visual Working Memory. The Journal of Neuroscience, 37(14), 3913-3925. https://doi.org/10.1523/JNEUROSCI.3493-16. 2017

Serences, J. T. (2016). Neural mechanisms of information storage in visual short-term memory. Vision Research, 128, 53-67. https:// doi.org/10.1016/j.visres.2016.09.010

Serences, J. T., Ester, E. F., Vogel, E. K., \& Awh, E. (2009). Stimulusspecific delay activity in human primary visual cortex. Psychological Science, 20(2), 207-214. https://doi.org/10.1111/j. 1467-9280.2009.02276.x

Sligte, I. G., Scholte, H. S., \& Lamme, V. A. (2008). Are there multiple visual short-term memory stores? PLoS One, 3(2), e1699. https:// doi.org/10.1371/journal.pone.0001699

Sligte, I. G., Scholte, H. S., \& Lamme, V. A. (2009). V4 activity predicts the strength of visual short-term memory representations. The Journal of Neuroscience, 29(23), 7432-7438. https://doi.org/10. 1523/JNEUROSCI.0784-09.2009

Sligte, I. G., Vandenbroucke, A. R., Scholte, H. S., \& Lamme, V. A. (2010). Detailed sensory memory, sloppy working memory. Frontiers in Psychology, 1, 175. https://doi.org/10.3389/fpsyg. 2010.00175 
Sligte, I. G., Wokke, M. E., Tesselaar, J. P., Scholte, H. S., \& Lamme, V. A. (2011). Magnetic stimulation of the dorsolateral prefrontal cortex dissociates fragile visual short-term memory from visual working memory. Neuropsychologia, 49(6), 1578-1588. https://doi.org/10. 1016/j.neuropsychologia.2010.12.010

Sreenivasan, K. K., Curtis, C. E., \& D'Esposito, M. (2014). Revisiting the role of persistent neural activity during working memory. Trends in Cognitive Sciences https://doi.org/10.1016/j.tics.2013.12.001

Tamber-Rosenau, B. J., Fintzi, A. R., \& Marois, R. (2015). Crowding in Visual Working Memory Reveals Its Spatial Resolution and the Nature of Its Representations. Psychological Science, 26(9), 15111521. https://doi.org/10.1177/0956797615592394

Todd, J. J., \& Marois, R. (2004). Capacity limit of visual short-term memory in human posterior parietal cortex. Nature, 428(6984), 751-754. https://doi.org/10.1038/Nature02466

Todd, J. J., \& Marois, R. (2005). Posterior parietal cortex activity predicts individual differences in visual short-term memory capacity. Cognitive, Affective, \& Behavioral Neuroscience, 5(2), 144-155.

Toet, A., \& Levi, D. M. (1992). The two-dimensional shape of spatial interaction zones in the parafovea. Vision Research, 32(7), 13491357.

van Moorselaar, D., Olivers, C. N., Theeuwes, J., Lamme, V. A., \& Sligte, I. G. (2015). Forgotten but not gone: Retro-cue costs and benefits in a double-cueing paradigm suggest multiple states in visual short-term memory. Journal of Experimental Psychology. Learning, Memory, and Cognition, 41(6), 1755-1763. https://doi. org $/ 10.1037 / \mathrm{xlm} 0000124$
Vandenbroucke, A. R., Sligte, I. G., \& Lamme, V. A. (2011). Manipulations of attention dissociate fragile visual short-term memory from visual working memory. Neuropsychologia, 49(6), 15591568. https://doi.org/10.1016/j.neuropsychologia.2010.12.044

Vandenbroucke, A. R., Sligte, I. G., de Vries, J. G., Cohen, M. X., \& Lamme, V. A. (2015). Neural Correlates of Visual Short-term Memory Dissociate between Fragile and Working Memory Representations. Journal of Cognitive Neuroscience, 27(12), 24772490. https://doi.org/10.1162/jocn_a_00870

Vogel, E. K., \& Machizawa, M. G. (2004). Neural activity predicts individual differences in visual working memory capacity. Nature, 428(6984), 748-751.

Whitney, D., \& Levi, D. M. (2011). Visual crowding: a fundamental limit on conscious perception and object recognition. Trends in Cognitive Sciences, 15(4), 160-168. https://doi.org/10.1016/j.tics.2011.02.005

Yoruk, H., \& Tamber-Rosenau, B. (2019). Reevaluating the sensory recruitment model of visual working memory using crowding anisotropy. Paper presented at the Workshop on Object Perception, Attention, and Memory, Montreal, Quebec.

Zhang, W., \& Luck, S. J. (2008). Discrete fixed-resolution representations in visual working memory. Nature, 453(7192), 233-235. https://doi.org/10.1038/nature06860

Publisher's note Springer Nature remains neutral with regard to jurisdictional claims in published maps and institutional affiliations. 